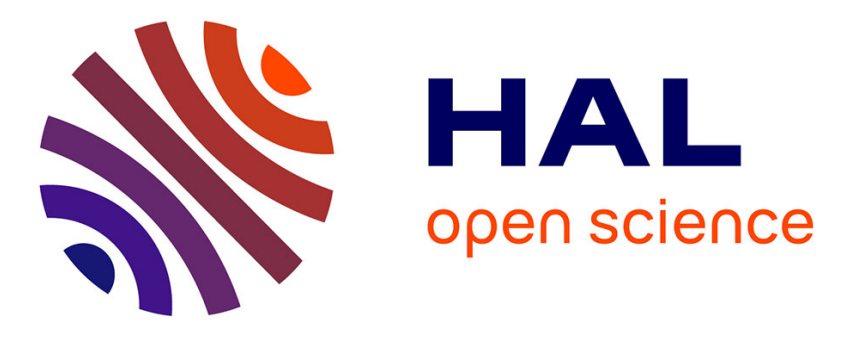

\title{
Driver Model Validation through Interaction with Varying Levels of Haptic Guidance
}

Yishen Zhao, Béatrice Pano, Philippe Chevrel, Fabien Claveau, Franck Mars

\section{To cite this version:}

Yishen Zhao, Béatrice Pano, Philippe Chevrel, Fabien Claveau, Franck Mars. Driver Model Validation through Interaction with Varying Levels of Haptic Guidance. SMC 2020: IEEE International Conference on Systems, Man, and Cybernetics, Oct 2020, Toronto, Canada. 10.1109/SMC42975.2020.9283382 . hal-02929636

\section{HAL Id: hal-02929636 https://hal.science/hal-02929636}

Submitted on 3 Sep 2020

HAL is a multi-disciplinary open access archive for the deposit and dissemination of scientific research documents, whether they are published or not. The documents may come from teaching and research institutions in France or abroad, or from public or private research centers.
L'archive ouverte pluridisciplinaire HAL, est destinée au dépôt et à la diffusion de documents scientifiques de niveau recherche, publiés ou non, émanant des établissements d'enseignement et de recherche français ou étrangers, des laboratoires publics ou privés. 


\title{
Driver Model Validation through Interaction with Varying Levels of Haptic Guidance
}

\author{
Yishen Zhao ${ }^{1}$, Béatrice Pano ${ }^{1}$, Philippe Chevrel ${ }^{1}$, Fabien Claveau ${ }^{1}$ and Franck Mars ${ }^{2}$
}

\begin{abstract}
Driver modeling is essential in the development of haptic guidance systems. A new cybernetic driver model designed to account for the cooperation between the driver and haptic guidance systems has recently been proposed. This paper aims to validate this model in situations of interaction with different levels of haptic guidance on a driving simulator. Two experiments have been performed for this purpose. The first experiment consisted of implementing the driver model in the driving simulator and evaluating its lateral control performance when interacting with a haptic guidance system. The results reveal that the model can be adapted to different sharing levels by adjusting only the gain of an internal model of the steering wheel compliance. The second experiment consisted of estimating the evolution of the gain of this internal model using the unscented Kalman filter. The results reveal consistency between the evolution of the identified parameter and the level of sharing of the haptic guidance system. The driver model represents the process of human driver adaptation to variations in the level of sharing in haptic guidance systems.

Index Terms - driver model, internal model, haptic guidance system, haptic shared control, adaptation, parameter identification
\end{abstract}

\section{INTRODUCTION}

In line with the increasing interest in research on advanced driver assistance systems, a mode of interaction between humans and automation called haptic shared control [1], [2] has been intensively studied. Haptic shared control proposes that the human driver and automation simultaneously apply a control action on an interface. In this case, the driver receives continuous haptic feedback and is aware of the action performed by the automation. Haptic shared control is considered an efficient method to achieve smooth humanmachine cooperation, especially in automotive applications [3]. It has proven to be safer in emergency situations than traditional modes [4]-[6]. When haptic shared control is applied to the steering control task, the assistance system is generally referred to as a haptic guidance system. Fig. 1 illustrates how the steering task is performed in cooperation between the driver and the system.

To minimize the potential conflicts between drivers and haptic guidance systems, understanding and predicting driver behavior is essential. For the development of some systems, a driver model was included in the design strategy to predict the output torque of the human driver [7], [8]. In a recent

\footnotetext{
${ }^{1}$ Yishen Zhao, Béatrice Pano, Philippe Chevrel and Fabien Claveau are with Institut Mines-Télécom Atlantique, LS2N UMR CNRS 6004 (Laboratoire des Sciences du Numérique de Nantes), 44307 Nantes, France firstname. lastnamedls $2 n$. fr

${ }^{2}$ Franck Mars is with CNRS \& Centrale Nantes, LS2N UMR CNRS 6004 (Laboratoire des Sciences du Numérique de Nantes), 44321 Nantes, France franck.marsels 2 n. fr
}

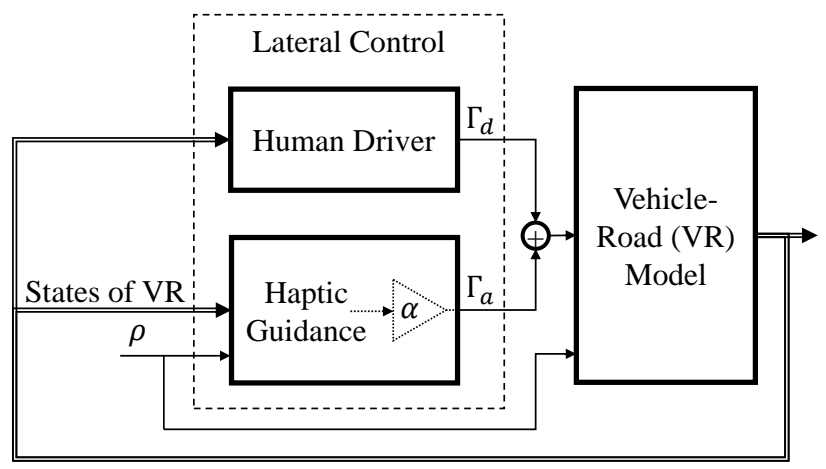

Fig. 1. Shared control between the human driver and haptic guidance system. $\rho$ : road curvature; $\alpha$ : sharing level; $\Gamma_{d}$ : driver steering torque; $\Gamma_{a}$ : haptic guidance torque.

study [9], a new cybernetic driver model was proposed. The advantage of this model is that it uses the haptic torque feedback as input to account for the cooperation between the driver and haptic guidance system. The model was identified under two conditions: manual driving and driving with a haptic guidance system whose sharing level was set at $50 \%$ (i.e., the system produced $50 \%$ of the total guidance torque, see Section III-B). Under both conditions, the model's prediction of steering torque control was accurate.

This article is a follow-up to our previous study. It aims to answer two questions related to the validation of the driver model: 1) Is the behavior of the model close to that of a human driver regardless of the level of sharing? 2) How can the model account for the driver's adaptation to variations in the level of sharing over the course of driving? Two experiments were conducted to answer these questions.

This article is organized as follows: Section II briefly reviews the driver model. Section III introduces the driving simulator and haptic guidance system used in the experiments of this study. Sections IV and V present the realization of the two experiments for validating the driver model: one by implementing the driver model in a driving simulator to evaluate its lateral control performance and another by continuously identifying model parameters. Finally, Section VI concludes the results and proposes our future work.

\section{Cybernetic Driver Model}

\section{A. Structure of the Proposed Cybernetic Model}

The proposed model structure is depicted in Fig. 2 with the description of signals listed in Table I. The basic hypothesis of the model is that, in steering control, the driver implicitly 
TABLE I

DESCRIPTION OF SIGNALS IN FIG. 2

\begin{tabular}{c|c}
\hline \hline Signal & Description \\
\hline$\theta_{f a r}$ & Far-point Angle \\
$\theta_{\text {near }}$ & Near-point Angle \\
$\delta_{d}$ & Target Steering-wheel Angle \\
$\delta_{S W}$ & Actual Steering-wheel Angle \\
$\Gamma_{I}$ & Torque from Internal Model \\
$\Gamma_{d}$ & Driver Steering Torque \\
$\Gamma_{f b}$ & Haptic Feedback Torque \\
$\Gamma_{a}$ & Haptic Guidance Torque \\
$\Gamma_{s}$ & Self-aligning Torque \\
\hline \hline
\end{tabular}

aims at a steering angle determined from the visual scene and then applies it to the steering wheel through the activation of muscles. The model structure reflects these two steps: 1) generating a target steering-wheel angle $\delta_{d}$ using a two-point visual model (the blue part) and 2) applying the target angle to a combination of limbs and the steering system through the neuromuscular system (NMS) and outputting a torque control (the orange and green parts). The two-point visual model takes two angles, a far-point angle and a near-point angle, as input to represent the visual information that the driver references. The haptic feedback torque $\Gamma_{f b}$, which is the combination of the haptic guidance torque and the selfaligning torque, is the key input to the neuromuscular model. It intervenes in the model in two ways. First, it implicitly influences an internal model of steering wheel compliance (dotted orange line). Second, it forms an explicit feedback loop (solid green line). One minimal realization of the model could be written as follows:

$$
\begin{aligned}
& \dot{x}(t)=A x(t)+B u(t) \\
& y(t)=C x(t)+D u(t)
\end{aligned}
$$

with

$$
\begin{aligned}
& y(t)=\Gamma_{d} \\
& u(t)=\left[\begin{array}{lll}
\theta_{f a r} & \theta_{\text {near }} & \Gamma_{f b}
\end{array}\right]^{T}, \\
& A=\left[\begin{array}{ccc}
-\frac{1}{T_{I}} & 0 & 0 \\
-K_{I} v_{x} \frac{1}{T_{N}} \frac{K_{c}}{v_{x}}\left(\frac{T_{L}}{T_{I}}-1\right) & -\frac{1}{T_{N}} & 0 \\
0 & -\frac{K_{f b}}{T_{f b}} & -\frac{1}{T_{f b}}
\end{array}\right] \\
& B=\left[\begin{array}{ccc}
0 & \frac{1}{T_{I}} & 0 \\
K_{I} v_{x} \frac{1}{T_{N}} K_{p} & K_{I} v_{x} \frac{1}{T_{N}} \frac{K_{c}}{v_{x}} \frac{T_{L}}{T_{I}} & 0 \\
0 & 0 & \frac{K_{f b}}{T_{f b}}
\end{array}\right] \\
& C=\left[\begin{array}{lll}
0 & 1 & 1
\end{array}\right] \\
& D=\left[\begin{array}{lll}
0 & 0 & 0
\end{array}\right]
\end{aligned}
$$

\section{B. Internal Model}

The internal model consists of a gain $K_{I}$ and a time constant $T_{N}$. In neural science, it is believed that the nervous system uses models of the physical world to exercise control and prediction to achieve skilled motor performance [10]. In the case of steering control, the driver uses an internal model to convert the target steering-wheel angle to torque. This internal model results from driver perception and prior knowledge of the steering system. During driving, the driver learns about the dynamics of the system and adapts to it by updating the internal model of steering system compliance. It is likely that this estimation is approximate and corresponds to a simple (low-order) model, which is represented by the gain $K_{I}$. From the driver's viewpoint, the dynamics of the steering system are assessed through the haptic feedback. The haptic feedback torque thus affects the internal model, which is represented by the dashed line in Fig. 2. The time constant $T_{N}$ represents the fact that the muscles need time to output the torque control.

\section{Explicit Haptic Feedback Loop}

To achieve the target steering-wheel angle and stable control of the steering wheel, an open-loop control through the internal model is not sufficient, as it is not able to compensate for any perturbations that arise during the movement. A closed-loop control using sensory feedback is indispensable. The explicit haptic feedback loop is a first-order system that outputs a complementary torque based on the error between the torque output of the internal model and the haptic feedback. This compensation through haptic feedback torque is considered faster and more intuitive for the driver than through the actual steering-wheel angle because small errors between the target and actual steering-wheel angle are more likely to be observed via visual information, and the compensation is therefore much longer. In other words, the $N M S$ is more likely to counterbalance the haptic feedback to stabilize the steering wheel than to achieve the exact target steering-wheel angle.

\section{EXPERIMENT SETTINGS}

\section{A. Apparatus}

The experiments in this study were all performed on a fixed-base driving simulator powered by SCANeR Studio (Fig. 3, left). The simulator is equipped with a complete dashboard; a five-speed gear stick; gas, brake, and clutch pedals; and a steering wheel connected to a TRW steering system. Sensors for measuring the steering-wheel angle, speed, and torque are mounted in the steering system. The visual scene is displayed on three liquid crystal display (LCD) screens: a central one in front of the driver and two others oriented at $45^{\circ}$ relative to the center. The screens cover a field of view of $25^{\circ}$ high and $115^{\circ}$ wide. The software allows a compilation and implementation of controllers developed in Simulink to control either the steering wheel or the vehicle. In all the experiments, a small family car, the Citroën C5, was chosen as the vehicle model, with the longitudinal speed fixed at $18 \mathrm{~m} / \mathrm{s}$. 


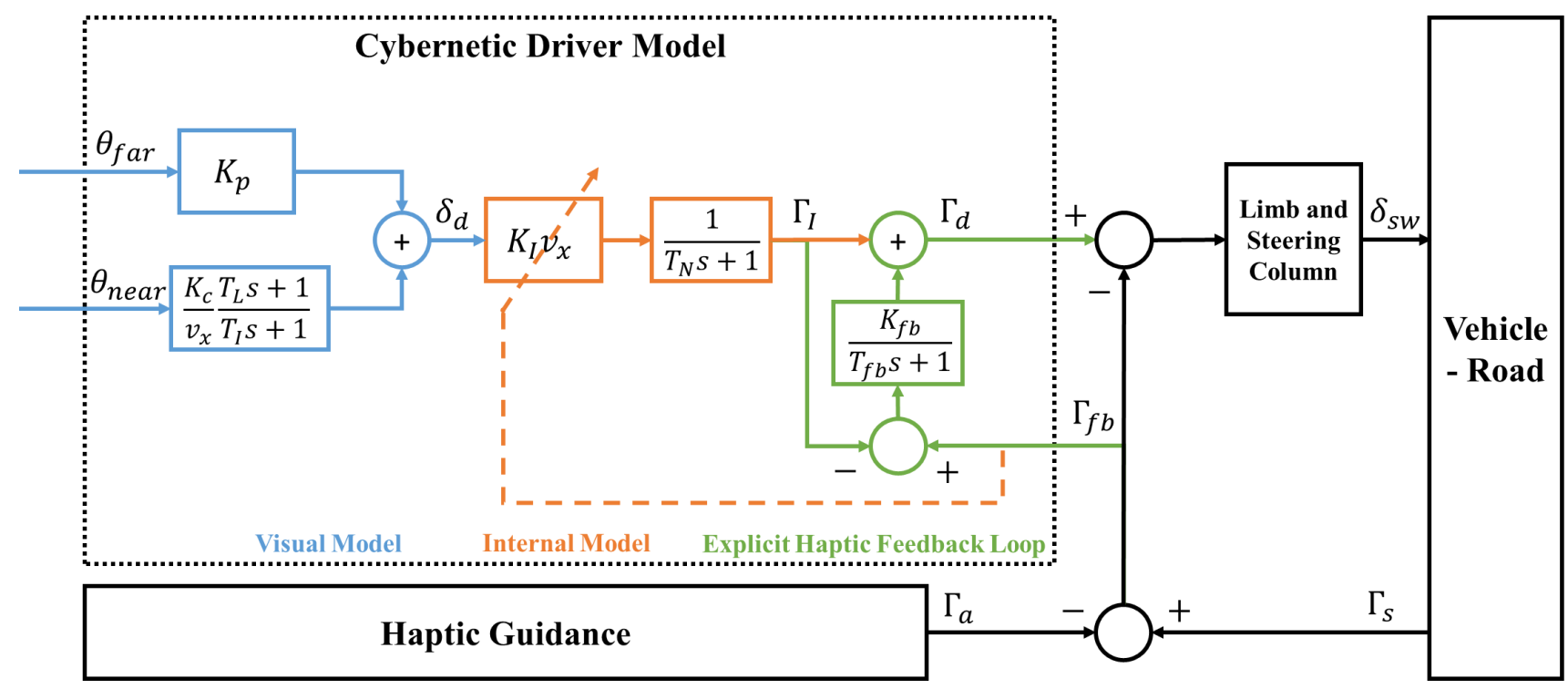

Fig. 2. Structure of the proposed cybernetic driver model. Blue: two-point visual model; Orange: driver internal model; Green: direct haptic feedback loop.
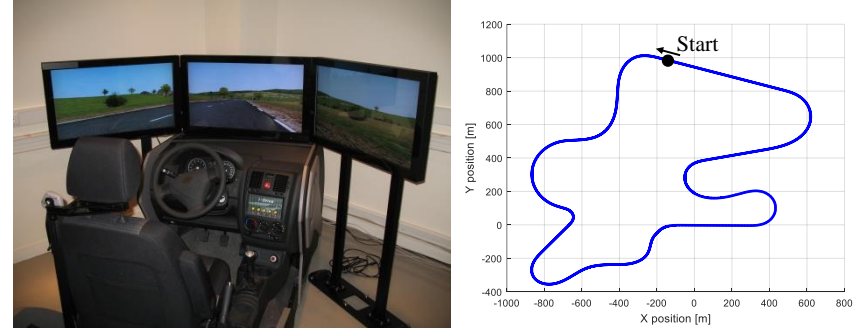

Fig. 3. Left: fixed-base driving simulator; Right: track used in the first experiment.

\section{B. Haptic Guidance System}

A haptic guidance system was implemented in the driving simulator using a controller designed in Simulink. The controller was previously developed [8]. The final guidance torque $\Gamma_{a}$ was a combination of an anticipatory and compensatory assistance torque depending on a factor $\alpha$ called the sharing level:

$$
\Gamma_{a}=\alpha \times \Gamma_{a n t}+\Gamma_{c o m p}(\alpha)
$$

where $\Gamma_{\text {ant }}$ and $\Gamma_{\text {comp }}$ are the torque generated by the anticipatory and compensatory assistance, respectively. The anticipatory assistance generates a reference trajectory from the previewed road curvature. The compensatory assistance consists of an $\mathrm{H}_{2} / \mathrm{H}_{\infty}$ static output feedback that controls the steering against disturbances and uncertainties. The sharing level determines how much guidance torque is finally applied on the steering wheel, and its value could vary from $0 \%$ (completely manual driving) to $100 \%$ (completely automatic driving).

\section{VAlidation I: Driver Model Simulation}

\section{A. Objective}

In a previous study [9], the parameters of the driver model were sequentially estimated using the prediction error minimization method [11] with driving data from five participants. Two sets of parameters were obtained under two experimental conditions: one for fully manual driving and one for driving with a haptic guidance system with a $50 \%$ sharing level. The model was validated under both conditions with a good prediction quality of driver torque control ( FIT between $85 \%$ and $92 \%$ ). The comparison between the two sets of parameters implies that the $N M S$, in particular, the internal gain $K_{I}$ of the model, accounts for the driver's adaptation to the intervention of the haptic guidance system. This observation corresponds to the expected role of the internal model in the model. Further, whether the driver's model can cooperate effectively with a haptic guidance system as soon as the gain of the internal model corresponds to the chosen sharing level remains to be verified.

\section{B. Experiment}

Fig. 4 illustrates how the experiment was conducted. Instead of a human driver, the driver model was implemented as a controller in the driving simulator to control the vehicle in cooperation with the haptic guidance system. Two independent variables were manipulated in this experiment: the sharing level of the haptic guidance system, $\alpha$, and the internal model gain, $K_{I}$. Four values for the sharing level were chosen: $0 \%, 30 \%, 50 \%$, and $80 \%$. The corresponding values of the internal model gain are $0.23,0.17,0.13$, and 0.04 . The other parameters in the driver model were fixed at their nominal values (Table II). The combination of the two independent variables provides 16 different experimental scenarios. Each scenario lasted for about $5 \mathrm{~min}$. A normal 


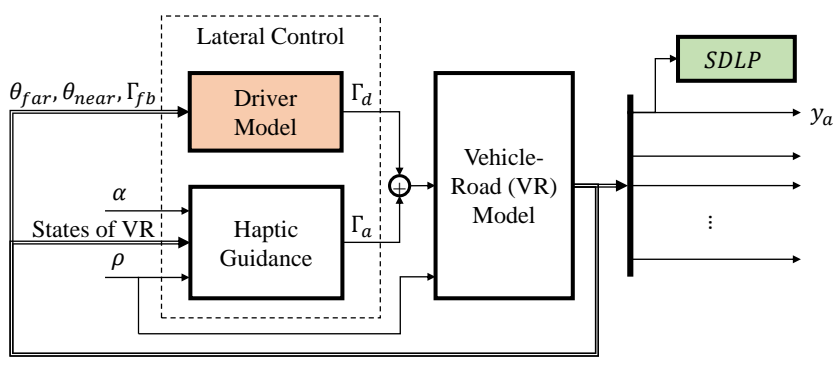

Fig. 4. Driver model validation with the implemented driver model.

TABLE II

Nominal Parameter VAlues USEd in Validation I

\begin{tabular}{c|c|c}
\hline \hline Parameter & Description & Value \\
\hline$K_{p}$ & Anticipatory Gain & 3.7 \\
$K_{c}$ & Compensatory Gain & 2.6 \\
$T_{I}$ & Compensation Time Constant & 0.4 \\
$T_{L}$ & Compensation Rate & 7.2 \\
$T_{N}$ & Neuromuscular Time Constant & 0.23 \\
$K_{f b}$ & Haptic Feedback Loop Gain & 0.97 \\
$T_{f b}$ & Haptic Feedback Loop Time Constant & 0.023 \\
$v_{x}$ & Vehicle Longitudinal Speed & $18(\mathrm{~m} / \mathrm{s})$ \\
\hline \hline
\end{tabular}

track with the Euler spiral turns and straight lines was chosen for this experiment (Fig. 3, right). The vehicle trajectory was recorded and used to evaluate the lateral control performance.

\section{Results}

The standard deviation of the lateral position (SDLP) from the lane center was chosen as the metric in this experiment to present the validation results. It is calculated using the lateral position deviation signal $y_{a}$, which is the distance from the center of gravity of the vehicle to the lane center (in meters). A relatively large $S D L P$ indicates poor lane-keeping performance.

The $S D L P$ values for all 16 scenarios are listed in Table III. Each $S D L P$ value on the diagonal is the lowest in its row and column. This implies that, when the sharing level and internal model gain match each other, the driver model and haptic guidance system reach a relatively stable cooperation with only a small variation in the lateral position. Note that the $S D L P$ is slightly higher for $\alpha=0$ than in the other three cases because the driver model in manual mode tends to cut corners, while the haptic guidance system tends to follow the center of the lane. For a given sharing level, either a larger or smaller value of the internal model gain results in a higher $S D L P$. In these situations, the torque output from the internal model is either too large or too small. Such bias on the internal model, which represents the compliance of the steering system, cannot entirely be compensated for by the explicit haptic feedback loop, which eventually leads to a difference between the target and actual steering-wheel angle. Therefore, the vehicle position oscillates between the left and right border (or even outside) of the lane.

In conclusion, the results indicate that the adjustment
TABLE III

Standard Deviation of the Lateral Position of All 16 SCENARIOS

\begin{tabular}{c|cccc}
\hline \hline & $K_{I}=0.23$ & $K_{I}=0.17$ & $K_{I}=0.13$ & $K_{I}=0.04$ \\
\hline$\alpha=0 \%$ & 0.51 & 2.39 & 5.47 & $33.03^{*}$ \\
$\alpha=30 \%$ & 0.69 & 0.23 & 0.46 & 1.75 \\
$\alpha=50 \%$ & 0.91 & 0.48 & 0.20 & 0.85 \\
$\alpha=80 \%$ & 1.14 & 0.81 & 0.55 & 0.20 \\
\hline \hline
\end{tabular}

* The scenario ended exceptionally due to the vehicle left completely the lane without return.

of the internal model gain through the haptic feedback is imperative when the driver model interacts with the haptic guidance system at different sharing levels. This adjustment is sufficient for a significant improvement in lateral control performance.

\section{VAlidation II: DRIVER MOdEl IDENTIFICATION}

\section{A. Objective}

The previous validation experience has shown that the adjustment of the internal model gain accounts for the adaptation of the driver model to various levels of sharing. The second experimental validation seeks to capture the adaptation of the human driver to the haptic guidance system through time-varying identification of the internal model gain. This is done in a situation in which the sharing level varies during driving from $0 \%$ to $100 \%$. The assumption is that the gain of the internal model varies homogeneously with the sharing level.

\section{B. Experiment}

Fig. 5 illustrates how the second experiment was conducted. The driver was instructed to steer the vehicle in cooperation with the haptic guidance system on the track shown in Fig. 6, a road consisting only of Euler spiral bends with a radius of curvature varying between $75 \mathrm{~m}$ and $95 \mathrm{~m}$. The experiment started in manual driving $(\alpha=0)$. After the vehicle passed $\mathrm{P} 1$, the sharing level started to increase from $0 \%$ to $100 \%$ in $50 \mathrm{~s}$. The transition was indicated on the simulator screen by a progress bar. When driving was fully automated, the driver was required to remove the driver's hands from the steering wheel. When the vehicle passed P2 on the road, a takeover request was issued and the sharing level began to decrease from $100 \%$ to $0 \%$ in $50 \mathrm{~s}$. The driving session lasted approximately $4 \mathrm{~min}$. The signals used to calculate the inputs and outputs of the driver model were recorded throughout the drive at $100 \mathrm{~Hz}$.

A group of 11 males and six females aged 24 to 53 (mean $=31$ years, standard deviation $=8.2$ years) participated in the experiment. All participants had a valid driver's license and drove regularly (12550 km per year on average). The participants had no known medical conditions that could affect their driving skills and had normal or corrected vision. None of them had ever experienced a haptic guidance system. 


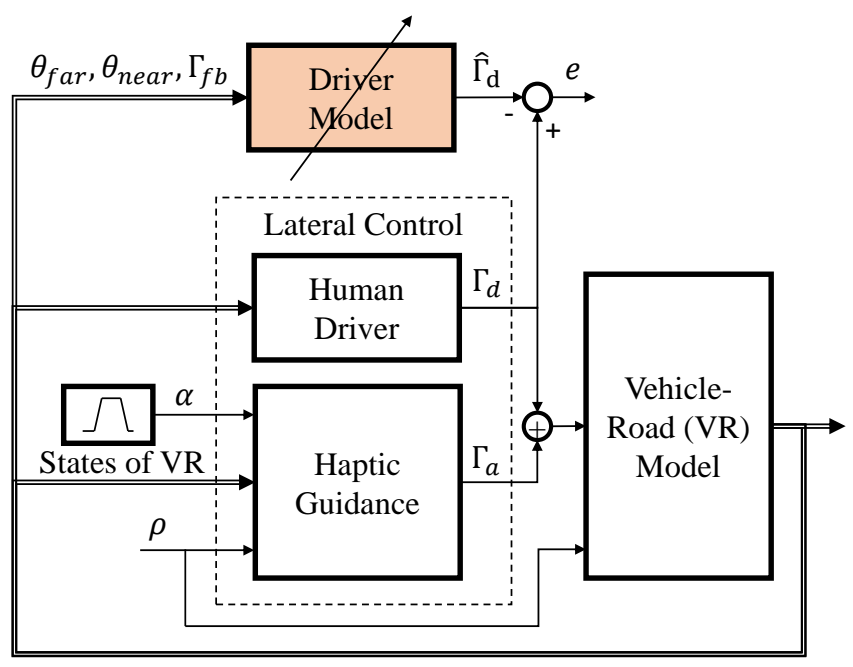

Fig. 5. Driver model validation with the identified driver model.

\section{Parameter Identification}

For each participant, the driver model identification was performed in two steps: 1) identification of the complete driver model using the data from the first manual driving phase (i.e., when $\alpha=0$ from the start of the experiment) and 2) estimation of the variation of the internal model gain $K_{I}$ using all the data in the experiment. The goal of the first step was to obtain an initial value for $K_{I}$ and a nominal value for the other parameters, which were needed in the second step. The identification method in the first step was the same as in [9]: the visual model, internal model, and explicit haptic feedback loop were identified sequentially by the prediction error minimization method. In the second step, as the value of $K_{I}$ may change continuously, a linear timevarying system identification method was used. The method was developed and validated in [12].

With the hypothesis that the variation of the internal model gain $K_{I}(t)$ can be modeled as a Wiener process and that it is slower than the variation of the system states, the system can be augmented with $K_{I}$ and then can be discretized using the Euler method. The augmented system derived from (1) in discrete time is written as follows:

$$
\left\{\begin{aligned}
x_{a}[k+1] & =f_{d}\left(x_{a}[k], u[k]\right)+w_{a, d}[k] \\
y[k] & =g_{d}\left(x_{a}[k], u[k]\right)+v[k]
\end{aligned}\right.
$$

with

$$
\begin{aligned}
& x_{a}[k]=\left[\begin{array}{c}
x[k] \\
K_{I}[k]
\end{array}\right]=\left[\begin{array}{c}
x\left(k T_{s}\right) \\
K_{I}\left(k T_{s}\right)
\end{array}\right] \\
& u[k]=u\left(k T_{s}\right) \\
& y[k]=y\left(k T_{s}\right) \\
& f_{d}\left(x_{a}[k], u[k]\right) \\
& =\left[\begin{array}{c}
\left.\left(T_{s} A\left(K_{I}[k]\right)+I\right) x[k]+T_{s} B\left(K_{I}[k]\right) u[k]\right] \\
K_{I}[k]
\end{array}\right. \\
& g_{d}\left(x_{a}[k], u[k]\right)=C x[k]+D u[k]
\end{aligned}
$$

where $w_{a, d}[k]$ and $v[k]$ are the discretized augmented process noise and measurement noise, with the covariance matrices

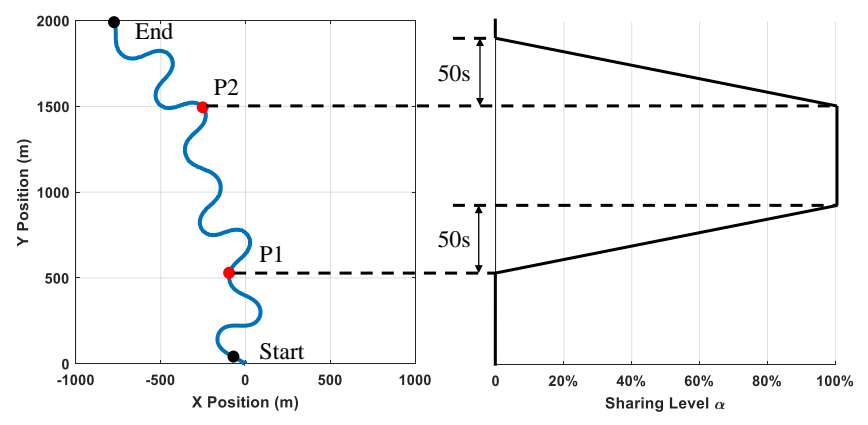

Fig. 6. Left: track used in the validation II; Right: variation of the sharing level in the experiment.

denoted as $Q_{a, d}$ and $R_{d}$, respectively. The $T_{s}$ is the sampling time, which was $0.01 \mathrm{~s}$ in this experiment. The $I$ in (6) is an identity matrix that is the same size as the matrix $A$. Note that the matrices $A$ and $B$ are all functions of $K_{I}$ in this case; thus in (6) the values evaluated at $K_{I}[k]$ are used.

The unscented Kalman filter (UKF) is applied to estimate the augmented system states $x_{a}[k]$ recursively by minimizing the following cost function:

$$
\begin{aligned}
J\left(\hat{x}_{a}[k]\right) & =\hat{x}_{a}^{T}[0] P_{a}^{-1}[0] \hat{x}_{a}[0] \\
& +T_{s} \sum_{k=0}^{N-1} \hat{w}_{a, d}^{T}[k] Q_{a, d}^{-1} \hat{w}_{a, d}[k] \\
& +T_{s} \sum_{k=0}^{N-1} \hat{v}_{d}^{T}[k] R_{d}^{-1} \hat{v}_{d}[k]
\end{aligned}
$$

where $P_{a}[0]$ is the initial covariance matrix of the augmented states and

$$
\begin{aligned}
\hat{w}_{a, d}[k] & =\hat{x}_{a}[k+1]-f_{d}\left(\hat{x}_{a}[k], u[k]\right) \\
\hat{v}_{d}[k] & =y[k]-g_{d}\left(\hat{x}_{a}[k], u[k]\right)
\end{aligned}
$$

The calculation steps of $U K F$ are listed in Algorithm 1.

The tuning methodology proposed by [12] was adopted to configure the filter, especially the value of the matrices $Q_{a, d}$ and $R_{d}$. The configuration of the filter demands a compromise between the rapidity and the precision (noise sensitivity) in the process of the estimation. A multi-model $U K F$ approach was finally applied to estimate simultaneously the steady-state value of $K_{I}$ and detect fast parametric variation with one relatively slow and another relatively fast filter.

\section{Results}

The FIT values were first obtained from the results of $U K F$ to verify the prediction of the driver torque. The calculation is as follows:

$$
F I T=\left(1-\frac{\left\|\Gamma_{d}-\hat{\Gamma}_{d}\right\|_{2}}{\left\|\Gamma_{d}-\operatorname{mean}\left(\Gamma_{d}\right)\right\|_{2}}\right) \times 100 \%
$$

where $\Gamma_{d}$ is the measured driver torque, and $\hat{\Gamma}_{d}$ is the prediction. For the slow UKF, the mean value of FIT for all participants is $91.5 \%$ with a standard deviation of $0.3 \%$. For the fast $U K F$, the mean value of FIT is $92.1 \%$ with a 
Algorithm 1 Estimation of $x_{a}[k]$ with the unscented Kalman
filter.

Step 1: Initialization

At time step $k=0$, configure $x_{a}[0], P_{a}[0], Q_{a, d}$ and $R_{d}$ :

Step 2: Calculation of the output prediction

At time step $k>0$, estimate the output prediction using the unscented transformation (UT) [13]:

$$
\begin{aligned}
& \left(\hat{y}[k \mid k-1], C_{y y}^{\prime}[k], C_{x y}[k]\right) \\
& =U T\left(g_{d}, \hat{x}_{a}[k \mid k-1], P_{a}[k \mid k-1]\right) \\
& C_{y y}[k]=C_{y y}^{\prime}[k]+R_{d}
\end{aligned}
$$

Step 3: Correction with measurements

At time step $k$, correct the values and the covariance of the augmented states with the measurements:

$$
\begin{aligned}
& * L[k]=C_{x y}[k] C_{y y}^{-1}[k] \\
& \hat{x}_{a}[k \mid k]=\hat{x}_{a}[k \mid k-1]+L[k](y[k]-\hat{y}[k \mid k-1]) \\
& P_{a}[k \mid k]=P_{a}[k \mid k-1]-L[k] C_{x y}^{T}[k]
\end{aligned}
$$

Step 4: States prediction for the next time step

At time step $k$, predict the augmented states value and covariance for the next time step $k+1$ using $U T$ :

$$
\begin{aligned}
& \left(\hat{x}_{a}[k+1 \mid k], P_{a}^{\prime}[k+1 \mid k]\right) \\
& =U T\left(f_{d}, \hat{x}_{a}[k \mid k], P_{a}[k \mid k]\right) \\
& P_{a}[k+1 \mid k]=P_{a}^{\prime}[k+1 \mid k]+Q_{a, d}
\end{aligned}
$$

Step 5: $k \leftarrow k+1$, repeat step 2 to 4 until $k=N$.
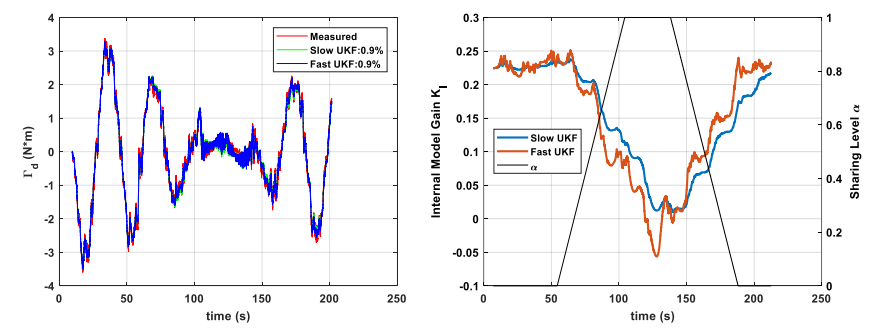

Fig. 7. Left: measured vs. predicted driver torque of Participant 1; Right: estimated $K_{I}$ variation of Participant 5.

standard deviation of $0.4 \%$. These values confirm the validity of the identified driver model. As an example, Fig. 7 (left) compares the prediction results of the Participant 1 . Note that, during the completely automatic driving phase (around $110 \mathrm{~s}$ to $150 \mathrm{~s}$ ), the driver torque was not exactly zero even though the participants did not touch the steering wheel. This was probably caused by the friction in the steering system that was measured by the torque sensor.

Second, the variation of $K_{I}$ was analyzed. Fig. 7 (right) presents the results of Participant 5 as an example. To check whether the variation of all participants is similar, the results were synchronized so that all participants passed Point P1 at the same time (after the synchronization, they passed the Point P2 with a time difference of less than $0.5 \mathrm{~s}$, which could be ignored). Fig. 8 reveals two curves

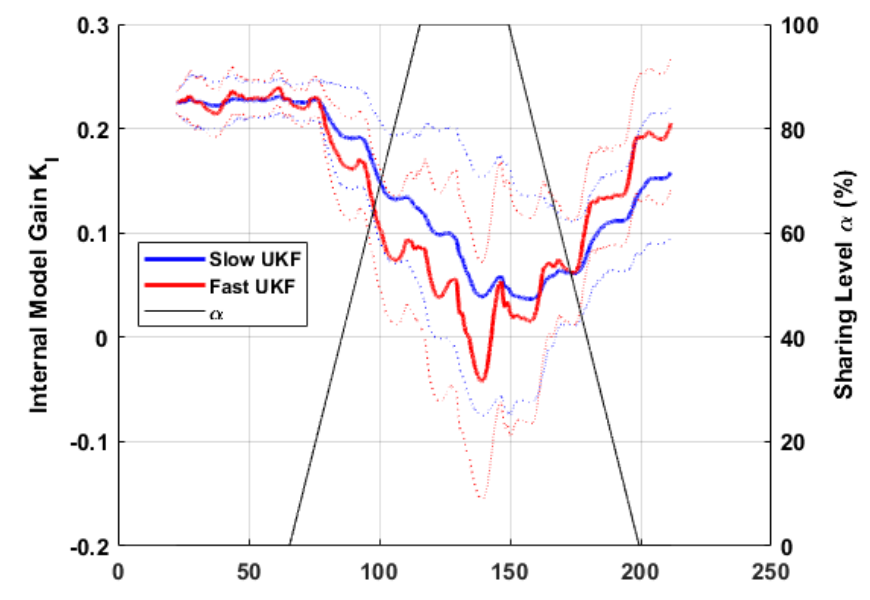

Fig. 8. Mean $K_{I}$ variation with the three-sigma band of all participants Blue: results of slow unscented Kalman filter(UKF); Red: results of fast $U K F$; Black: sharing level $\alpha$; Sold line: mean $K_{I}$; Dotted line: three-sigma band.

for the variation of mean $K_{I}$ with a three-sigma (three standard deviations) band of all participants, one estimated using the slow filter and another using the fast filter. The mean and standard deviation values were calculated with the estimated $K_{I}$ of all participants at each sampling instance using the synchronized results. The figure reveals that both the slow and fast filters exhibit a similar variation trend. For all participants, the internal model gain decreases when the sharing level increases, and vice versa. The $K_{I}$ is nearly zero during completely automatic driving. In addition, a delay of about $10 \mathrm{~s}$ occurs between the change in the sharing level and the variation of the internal model gain. During the experiment, human drivers adapted to the change of sharing level by delivering more or less torque on the steering wheel. This adaptation is captured very well by the variation of the internal model gain. In conclusion, the results demonstrated that the internal model gain of the driver model is directly related to the sharing level of the haptic guidance system. By adjusting this parameter, the prediction of the driver model in cooperation with the haptic guidance system is always valid when the sharing level changes.

\section{CONCLUSION}

The driver model proposed in [9] was developed to account for the cooperation between a driver and haptic guidance system. This paper aims to validate this model when the level of sharing between the two agents changes. Two experiments were conducted consecutively. The first experiment consisted of implementing the driver model in a driving simulator so that it could perform the steering task itself. The results indicated that the model that was identified for a given sharing level was no longer able to drive the simulator in cooperation with the haptic guidance system when the level of sharing changed. This implies that an adaptation of the model must occur either by changing the values of the parameters in the model or by changing the 
structure of the model.

The experiment demonstrates that this adaptation can be achieved by varying only the value of the gain of the internal model of the steering system compliance. As a corollary, this result validated the meaning attributed to the model parameters: the visual model is not affected by haptic guidance, and the neuromuscular time constant is invariant, as indicated in the literature [14]. Moreover, the explicit haptic feedback cannot perform the adaptation by itself. Ultimately, the adaptation of the internal model gain is imperative because, from the driver's viewpoint, the haptic guidance system modifies the dynamics of the steering wheel.

Based on these results, the second experiment validated the driver model with all possible sharing-level values. This time, the value of the internal model gain was identified using a $U K F$ method with driving data from human drivers who were experiencing slow transitions between manual and autonomous driving. This was achieved by gradually varying the sharing level. The results indicated that the variation in the internal model gain is directly related to the sharing level. This suggests that driver adaptation to the haptic guidance system is mostly achieved by updating the internal model. The driver model captures this adaptation process successfully.

Similar adaptation situations can be envisioned, such as when the driver discovers a new vehicle with a steering wheel that is more or less difficult to turn than expected. During the first few curves, lane control may be inaccurate and then improves as the next curves are negotiated. This adaptation would likely be done by adjusting the gain of the internal model; thus, the model would be able to account for it. This issue will be investigated in future studies.

\section{ACKNOWLEDGMENT}

This work was supported by RFI Atlanstic 2020, funded by Région Pays de la Loire, and AutoConduct research program funded by the French ANR "Agence Nationale de la Recherche" (grant ANR-16-CE22-0007-05).

\section{REFERENCES}

[1] M. Steele and R. B. Gillespie, "Shared Control between Human and Machine: Using a Haptic Steering Wheel to Aid in Land Vehicle Guidance," Proceedings of the Human Factors and Ergonomics Society Annual Meeting, vol. 45, no. 23, pp. 1671-1675, 2001.

[2] D. A. Abbink and M. Mulder, "Exploring the dimensions of haptic feedback support in manual control," Journal of Computing and Information Science in Engineering, vol. 9, no. 1, pp. 1-9, 2009.

[3] D. A. Abbink, M. Mulder, and E. R. Boer, "Haptic shared control: Smoothly shifting control authority?" Cognition, Technology and Work, vol. 14, no. 1, pp. 19-28, 2012.

[4] M. Mulder, D. A. Abbink, and E. R. Boer, "Sharing control with haptics: Seamless driver support from manual to automatic control," Human Factors, vol. 54, no. 5, pp. 786-798, 2012.

[5] P. G. Griffiths and R. B. Gillespie, "Sharing control between humans and automation using haptic interface: Primary and secondary task performance benefits," Human Factors, vol. 47, no. 3, pp. 574-590, 2005, pMID: 16435698.

[6] Z. Wang, R. Zheng, T. Kaizuka, K. Shimono, and K. Nakano, "The effect of a haptic guidance steering system on fatigue-related driver behavior," IEEE Transactions on Human-Machine Systems, vol. 47, no. 5, pp. 741-748, 2017.

[7] L. Saleh, P. Chevrel, F. Claveau, J. F. Lafay, and F. Mars, "Shared steering control between a driver and an automation: Stability in the presence of driver behavior uncertainty," IEEE Transactions on Intelligent Transportation Systems, vol. 14, no. 2, pp. 974-983, 2013.

[8] B. Pano, P. Chevrel, and F. Claveau, "Anticipatory and compensatory e-assistance for haptic shared control of the steering wheel," in 2019 18th European Control Conference (ECC), 2019, pp. 724-731.

[9] Y. Zhao, P. Chevrel, F. Claveau, and F. Mars, "Towards a Driver Model to Clarify Cooperation Between Drivers and Haptic Guidance Systems," submitted to 2020 IEEE International Conference on Systems, Man and Cybernetics (SMC).

[10] E. R. Kandel, J. H. J. H. Schwartz, T. M. Jessell, S. Siegelbaum, A. J. Hudspeth, and S. Mack, Principles of neural science, 5th ed. McGraw-Hill Education / Medical, 2013.

[11] L. Ljung, System Identification: Theory for the User, 2nd ed., ser. Prentice Hall information and system sciences series. Prentice Hall PTR, 1999.

[12] Y. Zhao, P. Chevrel, F. Claveau, and F. Mars, "Continuous Identification of Driver Model Parameters via the Unscented Kalman Filter," in 3rd IFAC Workshop on Linear Parameter Varying Systems, Eindhoven, Netherlands, 2019.

[13] S. J. Julier and J. K. Uhlmann, "New extension of the Kalman filter to nonlinear systems," in Proc. SPIE 3068,Signal Processing, Sensor Fusion, and Target Recognition VI, 1997.

[14] L. Saleh, P. Chevrel, F. Mars, J. F. Lafay, and F. Claveau, "Human-like cybernetic driver model for lane keeping," in Proceedings of the 18th IFAC World Congress, 2011, pp. 4368-4373. 